\title{
Metodología para valorar las a portaciones de la negociación en la gestión tecnológica de proyectos en un centro de I\&DT universitario
}

\author{
Valuation Methodology for the Intangible Negotiation Contributions to the \\ Projects Technology Management in a University R\&D Center \\ Vega-González L.R. \\ Coordinación de Vinculación y Gestión Tecnológica \\ Centro de Ciencias Aplicadas y Desarrollo Tecnológico (CCADET) \\ Universidad Nacional Autónoma de México \\ E-mail:1rvg@servidor.unam.mx
}

(Recibido: enero de 2009; aceptado: abril de 2010)

\begin{abstract}
Resumen
A partir del modelo de malla cerrada de circuitos de negociación en la gestión tecnológica de proyectos (GT de P); se plantea una metodología de valoración de las aportaciones intangibles de la negociación en la GT de P, basada en la definición y optimización de una función matemática de costos que debe cumplir con criterios específicos. Para su aplicación, se requiere que tanto los responsables de vinculación como el equipo de desarrollo del proyecto identifiquen y cuantifiquen los lazos o circuitos de negociación requeridos en el proyecto. Para disminuir la complejidad del problema, se presentan algunos criterios de valoración, que a su vez, dan el fundamento de la metodología. La GT de P no se aplica a todos los proyectos de investigación aplicada y desarrollo tecnológico (I\&DT), que realiza una organización; sin embargo, es claro que en todos ellos se lleva a cabo al menos un circuito de negociación para lograr la aceptación de la propuesta técnico-económica. Al final de este trabajo, se propone una función que relaciona el rango óptimo de probabilidades de éxito con respecto al porcentaje de proyectos en los que se aplica negociación en las diferentes etapas de la GT de P. La metodología presentada permite calcular el valor que pueden tener las aportaciones de la negociación respecto al valor total del proyecto.
\end{abstract}

Descriptores: valoración de intangibles, circuitos de negociación, gestión tecnológica de proyectos.

\footnotetext{
Abstract

A valuation methodology for the intangible negotiation contributions is proposed from the negotiation circuits of the Management of Technology Development Projects (MTDP) and linking model. It is based in the definition and optimization of specific criteria mathematical cost function. The application of the methodology requires the identification of a the negotiation circuits required for the project by the development team and the technology transfer and responsible linking office. To reduce the problem complexity some valuation criteria are used as the methodology fundaments. The MTDP model activities are not
} 
DOI: http://dx.doi.org/10.22201/fi.25940732e.2010.11n4.032

Metodología para valorar las aportaciones de la negociación en la gestión tecnológica de proyectos en un centro de I\&DT universitario

applied to all the organization's R\&D projects, but it is clear that in all of them it is at least required one negotiation circuit to get the acceptance of the technical and economical project proposals. Finally, a function that covers the relation of the optimal probability success with respect to the percentage of those projects using negotiation at the different phases of the MTDP model is proposed.

The methodology presented allows the calculation of the negotiation contributions with respect to the whole Project value.

Keywords: Intangible negotiation valuation, project management of technology, University-Enterprise linking.

\section{Descripción del problema}

A mediados del siglo XVII los economistas clásicos Adam Smith y David Ricardo hicieron los planteamientos de "la mano invisible" y del libre mercado, como motores de la economía y la fuente primaria de la riqueza de las naciones (Tribe, 2006). A mediados del siglo XVIII, con la disponibilidad de maquinaria y el control del vapor como fuente primaria de energía, otros pensadores atribuyeron la riqueza a la creación de valor por medio de la manufactura y la transformación de las mercancías realizadas por los trabajadores; con lo que surgió el concepto de la apropiación y acumulación del capital. Por más de un siglo la tendencia fue la optimización de los sistemas de productivos, mientras que la fuente de la riqueza se ligaba con la propiedad de los medios o tecnologías para la producción. La consecuencia natural fue el desarrollo de tecnologías de proceso, producto, equipamiento y las tecnologías "blandas" como las operacionales. Con esta proliferación tecnológi$\mathrm{ca}$, vino el acortamiento del ciclo de vida de las tecnologías y una era de "discontinuidad" (Drucker, 1992). Como consecuencia, en los años ochenta del siglo pasado, se entendió que la riqueza de las naciones dependía de que sus organizaciones productivas impulsaran el dominio tecnológico, como la fuente de su ventaja competitiva (Porter, 1997). Una década después se planteó que la fuente de la riqueza provenía de la capacidad de innovación de las organizaciones (Drucker, 2002). Hoy en día, este concepto está plenamente rebasado y se habla de sistemas de gestión tecnológica integrados (Geistauts et al., 1992) y de la gestión del conocimiento (Ching, 2000). En todo caso, desde el punto de vista sistémico, el concepto holístico actual es el de que la riqueza de una nación depende del nivel de integración y actuación de su Sistema Nacional de Innovación (SNI).

Según Lundvall (1992), las tres características fundamentales de un SNI son: a) La generación de nuevo conocimiento,

b) La capacidad de absorción de éste, por parte de todas las organizaciones o individuos que la conforman, es decir, la capacidad de asimilación tecnológica,

c) Que no exista un ambiente perjudicial a la misma.

En este marco, y en nuestro contexto, hoy en día las universidades, las escuelas tecnológicas y en particular, los institutos y centros de I\&DT, tanto públicos como privados, tienen una gran responsabilidad, ya que deben ser fundamentalmente generadores de nuevo conocimiento científico y tecnológico. En particular, para Hindle y Yencken (2004) el conocimiento obtenido a través de la investigación pública debería comercializarse a través de nuevas empresas de base tecnológica a las que el nuevo conocimiento tendría que dar origen.

Hablando metafóricamente, el conocimiento científico-tecnológico es el combustible en el SNI, pero es indispensable el desarrollo de otras habilidades organizacionales que funcionen como lubricantes del sistema. Así, para poner a disposición de los usuarios finales el conocimiento tecnológico generado, se requiere que haya una Gestión Tecnológica efectiva.

A nivel internacional hay un reconocimiento creciente de que la GT es un elemento clave en el proceso de negocios y debe integrarse en el plan estratégico de la organización (Bone y Saxon, citados por Jacob \& Kwak, 2003). También hay que considerar que, debido a la disponibilidad creciente de nuevas tecnologías, la administración tecnológica ha incrementado su importancia y hoy se considera como una actividad crucial para las organizaciones industriales y gubernamentales (Linn et al., 2000).

Frecuentemente, en el ámbito de los centros de investigación y desarrollo tecnológico (I\&DT) de las universidades mexicanas, la unidad de trabajo es el proyecto, por lo que en términos más amplios la GT de proyectos, (GT de P) incluye la administración tecnológica de uno o varios proyectos. 
DOI: http://dx.doi.org/10.22201/fi.25940732e.2010.11n4.032

Vega-González L.R.

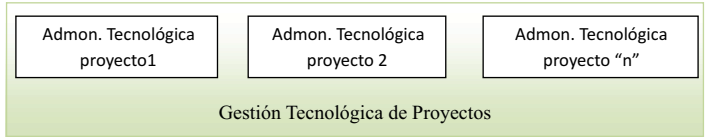

Figura 1. Relación entre la gestión tecnológica de proyectos y la administración tecnológica

Para el personal académico que realiza la I \&DT y a veces aún para los directivos, la necesidad y complejidad de la GT de P no es reconocida en su conjunto; menos aún se reconoce el valor de las negociaciones que se realizan dentro de las distintas etapas de la misma. Es por esta razón, que el primer paso para valorar las aportaciones de la negociación a la GT de P, es su reconocimiento y el entendimiento de que se trata de un intangible.

En términos concretos, el problema operativo que nos ocupa en este trabajo, incide en el procedimiento de costeo de los proyectos patrocinados que desarrolla un centro de I\&DT universitario, en el contexto ya mencionado de que el valor intangible de la negociación inmersa en la Gestión Tecnológica de Proyectos (GT de $\mathrm{P})$, no es visible ni evidente.

Cuando el personal académico científico ó técnico desarrolla propuestas, casi nunca considera los costos de gestión y negociación. Esto resulta evidente cuando se obtiene el contrato de un proyecto de $I \& D$ por parte de un patrocinador y se realiza el documento de control interno de apertura de proyecto. Frecuentemente se omite el costo de la participación de las áreas de vinculación y gestión tecnológica. Parece que muchos académicos sólo se apegan a los procedimientos de costeo clásico (Kerzner, 1989), Knoepfel, 1986) en el que, de acuerdo con los procedimientos estándar, sólo se deben considerar como Costos Directos (CD) los aspectos relacionados con el tiempo y los recursos humanos especializados requeridos para el desarrollo, así como los materiales, la información requerida y otros aspectos directamente relacionados con el desarrollo técnico del proyecto. Esta es una omisión, ya que la aportación de las diferentes actividades de la GT de P, incluyendo las propias de la gestación de proyectos, por su naturaleza requieren de plazos de tiempo mayores que los que toma el mismo desarrollo técnico del proyecto. De hecho, como puede verse en la figura 1, la GT de P incluye a la AT.

En muchos casos, ni en los directivos ni en los consejos de evaluación académica existe la idea intuitiva de que las actividades de GT de P aportan valor al mismo proyecto. De hecho, desde el punto de vista sistémico, la GT de $\mathrm{P}$ es una actividad altamente especializada, donde se conjuntan conocimientos técnicos, administrativos y legales, cuyo objetivo fundamental es la creación de valor. (Acosta, et. al., 2000).

\section{El Centro de Ciencias Aplicadas y Desarrollo Tecnológico (CCADET)}

El CCADET es una de las entidades de la UNAM que cuenta con una Coordinación de Vinculación y Gestión Tecnológica, creada para fortalecer la vinculación con las empresas y organismos patrocinadores externos. La misión del CCADET es realizar investigación original aplicada y desarrollar tecnología para procesos y productos innovadores que ayuden a resolver problemas de interés nacional (Lara, 2005). Esto indica la necesidad explícita de que exista un problema tecnológico bien identificado, hacia el cual orientar los esfuerzos de investigación y desarrollo. La misión expresa además, que se preferirán aquellos problemas que tengan mayor impacto social. En ese sentido, el CCADET ha adoptado la visión de Adams et al. (1998), quien indica que el desempeño en el desarrollo de nuevos productos tecnológicos mejora cuando se aplica una perspectiva de aprendizaje organizacional y se logra un entendimiento profundo de las necesidades y deseos del cliente.

La vinculación también forma parte de los objetivos del CCADET, ya que en ellos, se propone establecer los medios para transferir los desarrollos tecnológicos realizados a los sectores productivos a fin de contribuir con la innovación tecnológica nacional. De hecho, en un contexto más amplio, en el CCADET se entiende la transferencia tecnológica universitaria, en el sentido expresado por Medellín (1996), como la transmisión de conocimientos tecnológicos en cualquiera de sus formas.

Según Hidalgo (1997), en el mercado tecnológico la demanda le corresponde a las empresas y la oferta a la Universidad. La oferta tecnológica universitaria debe estar orientada al cliente; es decir, se prefieren problemas tecnológicos donde existe un demandante para realizar desarrollos e investigaciones. Por esta razón, a partir del año 2002, la Coordinación de Vinculación $(\mathrm{CV})$, del CCADET ha desarrollado programas de mercadeo y promoción para ofrecer su producto principal, que es la capacidad intelectual de sus miembros, por lo que se han requerido desarrollar técnicas y procedimientos para lograr una gestión tecnológica eficiente y un buen control de proyectos. La CV del CCADET es la encargada de realizar la GT de P en los casos que así se le requiera de parte de los laboratorios que conforman el Centro. De esta forma, realiza diversas actividades de gestión, tales como el establecimiento de 
DOI: http://dx.doi.org/10.22201/fi.25940732e.2010.11n4.032

Metodología para valorar las aportaciones de la negociación en la gestión tecnológica de proyectos en un centro de I\&DT universitario

estrategias de propiedad intelectual, la aplicación de metodologías de evaluación y avalúo, prospectiva y monitoreo tecnológicos, mercadotecnia y finanzas aplicadas a proyectos de desarrollo tecnológico, entre otras.

En el CCADET se intenta revalorar el papel de la gestión tecnológica y la necesidad de su aplicación en las distintas áreas de proyectos de investigación y desarrollo, así como revitalizar el papel del gestor tecnológi$\mathrm{co}$, que actúa como negociador o como gerente de proyectos.

\section{Metodología de valuación de las aportaciones de la GT de P}

La metodología de valuación del intangible que presentamos en este trabajo, propone que la cuantificación del valor relacionado con las actividades de GT de P está directamente relacionada, en principio, con el número de horas aplicadas por el especialista para aplicar las distintas técnicas de gestión. En particular, se puede determinar el número de rondas de negociación en las que se participa, así como la eficacia en la obtención de resultados.

La metodología de valuación resulta de la combinación del modelo simplificado de malla cerrada de la GT de $P$, mostrado en la figura 2 , en el que se muestran e identifican los distintos circuitos de negociación que se llevan a cabo en la GT de P típica y de la adaptación del modelo matemático de costos de proyectos de I\&D, desarrollado por Lieb (1998).

El modelo de Lieb fue concebido originalmente para determinar el número óptimo de proyectos de investigación que una institución de I\&DT debe desarrollar, con el objetivo de disminuir la incertidumbre propia de los desarrollos eventuales. Dado que el costo es el valor monetario de los bienes y servicios usados para generar un producto físico o intelectual (Knoepfel et al., 1986), la premisa fundamental del planteamiento que desarrollaremos a continuación, es que el número óptimo de proyectos que desarrolla la organización, debe ser una función de la efectividad del costo de los esfuerzos de I\&DT y de la habilidad de la organización para soportarlos.

El modelo se basa en el concepto de que la función de esfuerzos de I\&DT debe buscar mejorar el rango de desarrollos de éxito. Nuestra hipótesis es que la habilidad de la organización para soportar e impulsar los proyectos con efectividad y eficiencia, depende en parte de una buena GT de P.

El modelo deriva hacia la valuación de los costos intangibles de las actividades de I \&DT, ya que hay que considerar que son proyectos de riesgo porque existe un alto grado de incertidumbre, por lo que se requiere definir criterios de valoración y escalas de juicio que provean una base sólida para la toma de decisiones.

En primer lugar, supondremos que en un Centro de I\&DT no todos los proyectos de Desarrollo Tecnológico

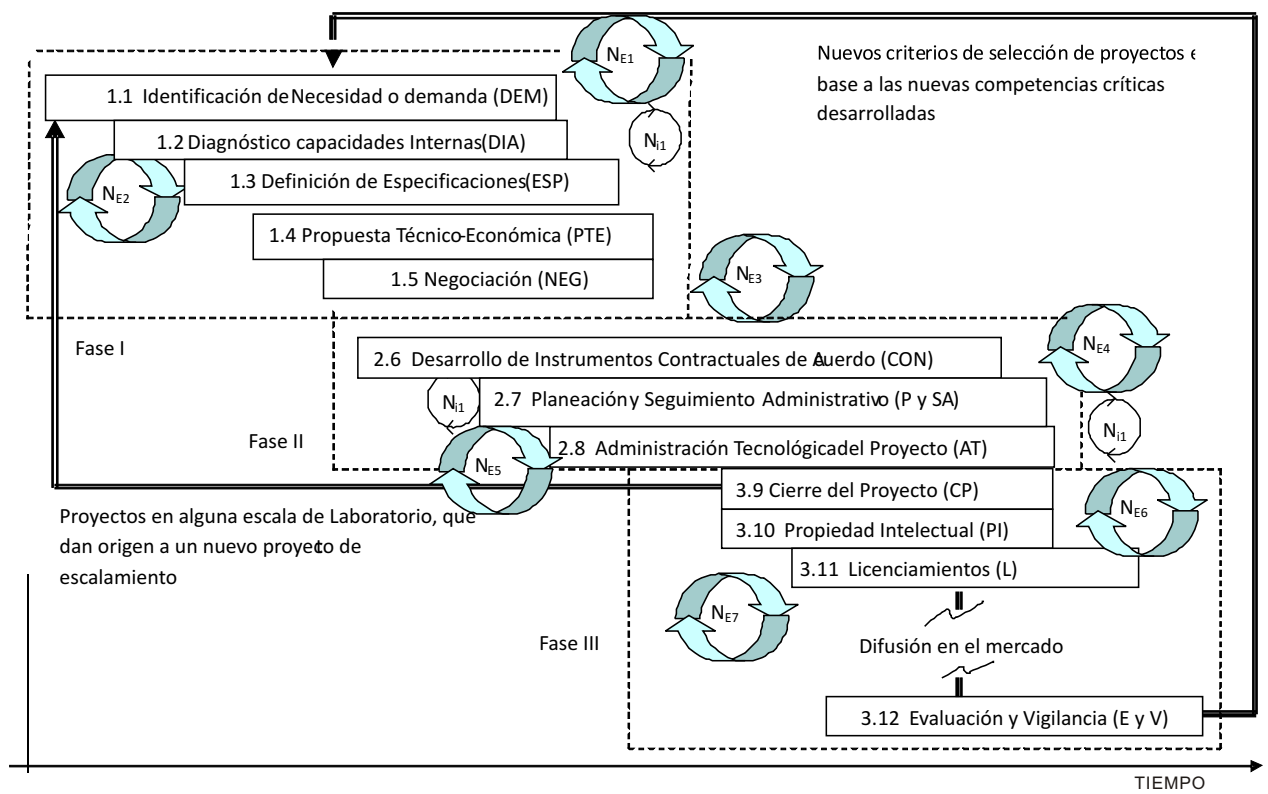

Figura 2. Modelo simplificado de malla cerrada de la GT de P, con la identificación de círculos de negociación 
DOI: http://dx.doi.org/10.22201/fi.25940732e.2010.11n4.032

Vega-González L.R.

(DT) que se llevan a cabo reciben apoyo de la Unidad de Vinculación y Gestión Tecnológica, especialmente en el área de gestión de la negociación. Entonces, trataremos de responder a la siguiente pregunta: ¿Cuál es el valor, en términos de costos, que aporta la actividad de Negociación al Costo total de los proyectos de DT? En otras palabras, si el costo de la negociación no se agrega al valor final de la tecnología representa un descuento para quien adquiere la tecnología.

A partir de nuestros resultados determinaremos cuál es el número más apropiado de proyectos a los que se puede aplicar la GT de P y en especial, las actividades recursivas de la negociación.

\section{Precios y costos de los proyectos}

Los centros de I\&DT producen conocimientos y soluciones tecnológicas, por lo que forman parte del mercado, en la economía del conocimiento. En ellos, por lo general, no se ostentan monopolios, por lo que al igual que cualquier otra organización, deben responder a la pregunta خ̇Cuál es el precio que permitirá ganar el proyecto? (Kerzner, 1989).

La estimación de precios es para muchos directivos y gerentes un arte. En el caso de proyectos I\&DT una buena estimación de precios requiere en principio de identificary sistematizar los requerimientos de materiales, de horas hombre, el tiempo de uso y la depreciación del equipamiento industrial, de laboratorio o científico; la valoración del conocimiento tácito y explícito de los científicos e ingenieros y de los costos de capital y organizacionales.

Así pues, una buena estimación de precios tiene su base en un buen planteamiento de costos. En el siguiente desarrollo, los costos de los circuitos de negociación en la GT de $\mathrm{P}$ son considerados como costos organizacionales.

El primer supuesto fundamental es que en una institución que realiza desarrollo tecnológico algunos proyectos reciben apoyo de gestión ${ }^{1}$ y otros no lo reciben, ya que la misma la realizan directamente los académicos, directivos u otras autoridades administrativas. Por otra parte, también existe una fracción de proyectos que reciben apoyo para su negociación.

La suma total de costos de proyectos de desarrollo (DT) es igual a la suma de Costos Directos (CD) de proyectos de DT que no reciben el apoyo de gestión tecnológica, más los que sí lo reciben.

$$
C D=(C g+C n g)
$$

\footnotetext{
${ }^{1}$ Normalmente para la gestión jurídica y algunas otras etapas del modelo presentado en la figura 2.
}

Donde:

$C g=$ Costo promedio de los proyectos que se gestionan

$C_{n g}=$ costo promedio de los proyectos que no se gestionan.

$C D=$ Costos directos promedio de todos los proyectos de desarrollo, los que reciben apoyo de gestión y los que no lo reciben.

Sí además se agrega la fracción de costos de aquellos proyectos $(\boldsymbol{x})$, que sí reciben apoyo de Negociación multiplicado por el número de proyectos en desarrollo $(\mathrm{N})$.

Entonces:

$$
C=N(C D+x C n)
$$

donde:

$C=$ costo total de proyectos de desarrollo tecnológico.

$\boldsymbol{x}=$ fracción de proyectos que reciben apoyo de negociación.

$C_{n}=$ costo promedio de los proyectos que reciben apoyo de negociación.

$N=$ número total de proyectos de desarrollo.

Por otra parte, sabemos que un proyecto puede considerarse exitoso cuando se entregan los productos acordados en tiempo y forma; esto es, de acuerdo al calendario propuesto inicialmente. Los entregables en general, pueden ser informes, dispositivos tecnológicos de hardware o software.

Al final, se obtiene una minuta o cartas de satisfacción del patrocinador y el pago acordado. Otra medida de éxito del proyecto es cuando no se rebasan los presupuestos considerados inicialmente y se puede identificar o medir el tipo de impacto económico, social, su trascendencia, etcétera.

Otra variable muy importante en el desarrollo del modelo es la medida del desempeño de la organización en la ejecución de los proyectos, por lo que definiremos $(\boldsymbol{y})$ al promedio de proyectos desarrollados exitosos.

Otro supuesto del modelo es que en la organización se desarrollarán un número suficiente de proyectos, de forma que al menos se obtenga en promedio un caso de éxito.

Esta consideración no limita el modelo, ya que los resultados serán proporcionales para cualquier número de casos. Entonces: 


$$
0<y \leq 1
$$

También buscamos que el número de proyectos exploratorios que se realicen sea igual al recíproco de la ocurrencia promedio para que el proyecto sea negociado y que a su vez sea exitoso.

$$
N=\frac{1}{x y}
$$

Por ejemplo, sí se gestionan y negocian un promedio de cinco de cada veinte proyectos que se desarrollan; es decir si $\mathrm{x}=5 / 20=0.25$; y si se sabe que el porcentaje promedio de proyectos de DT con éxito es del $50 \%$, esto es: $y=0.5$; entonces, el número de proyectos promedio que requiere desarrollar la organización será:

$$
N=\frac{1}{x y}=\frac{1}{(0.25)(0.5)}=8
$$

En otras palabras, bajo las condiciones señaladas, se requiere desarrollar 8 proyectos en los que apliquen las técnicas de Gestión y negociación para obtener un proyecto de éxito.

Con (4) en (2) la ecuación de costos se modifica:

$$
C=N(C D+x C n)=\left(\frac{1}{x y}\right)(C D+x C n)
$$

Ahora conviene definir el índice de la relación de los costos promedio de proyectos que reciben apoyo de negociación entre los costos directos promedio de todos los proyectos de DT, esto es:

$$
R=\frac{C n}{C D}
$$

Dividiendo (5) entre CD

$$
\frac{C}{C D}=\left(\frac{1}{x y}\right)\left(\frac{1}{C D}\right)(C D+x C n)=\frac{(1+R x)}{x y}
$$

\section{Rango y dominio de la función de costos}

Para propósitos de análisis, restringiremos la variación de la fracción de proyectos de DT exitosos $(\boldsymbol{y})$, entre dos valores límite: un valor máximo $(\beta)$ y un mínimo $(\alpha)$. Asimismo, la fracción de proyectos de DT a los que se realiza negociación tendrá la siguiente variación:

$$
0<x \leq 1
$$

Por razones de saturación de la organización, una función que resulta apropiada para graficar la distribución de la probabilidad de éxito contra la fracción de proyectos desarrollados es la mostrada en la figura 3.

\section{Relación $x-y$}

Para definir la relación existente entre la fracción de proyectos negociados y el porcentaje de proyectos exitosos, consideraremos que a mayor cantidad de proyectos de DT que realiza la organización en un periodo de tiempo determinado, habrá más proyectos negociados, pero por razones de saturación, en cuanto a la capacidad de desarrollo del personal académico responsable, se obtendrá un mínimo de proyectos de DT exitosos. Inversamente, a una menor fracción de proyectos negociados se obtiene una probabilidad mayor de éxito en los proyectos, debido a que los proyectos reciben más atención y los esfuerzos organizacionales se centran en un menor número de proyectos.

Una función matemática que responde a las consideraciones anteriores es la presentada en la ecuación (8), cuya gráfica se presenta en la figura 3.

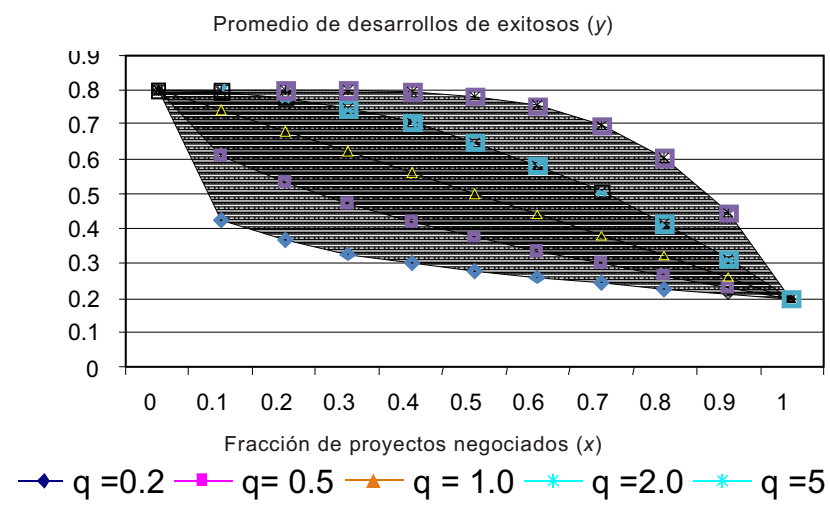

Figura 3. Probabilidad de proyectos de éxito en función de los proyectos negociados

$$
(\alpha-\beta) x^{q}+\beta
$$

Se trata de una familia de curvas planteada en términos de distintos valores del parámetro (q). Si el parámetro de calidad $q=1$, entonces el modelo es lineal:

$$
y=(\alpha-\beta) x+\beta
$$

Que es la ecuación de una recta con pendiente negativa. Los parámetros $\beta$ y $\alpha$ miden la ocurrencia de éxito máximo y mínimo, respectivamente. 
DOI: http://dx.doi.org/10.22201/fi.25940732e.2010.11n4.032

Vega-González L.R.

Sustituyendo (9) en (7):

$\frac{C}{C D}=\frac{(1+R x)}{x[(\alpha-\beta) x+\beta]}=\frac{(1+R x)}{\alpha x^{2}-\beta x^{2}+x \beta}$

Conviene ahora definir otro parámetro que siempre será positivo

$\Psi=\frac{(\beta-\alpha)}{\beta}=1-\frac{\alpha}{\beta}$

Entonces:

$\alpha=\beta-\beta \Psi$

Con (12) en (10)

$\frac{C}{C D}=\frac{(1+R x)}{\alpha x^{2}-\beta x^{2}+x \beta}=\frac{(1+R x)}{\beta x^{2}-\beta \Psi x^{2}-\beta x^{2}+\beta x}$

$\frac{C}{C D}=\frac{(1+R x)}{\beta\left(-\Psi x^{2}+x\right)}$

La ecuación (13) es la razón de costos totales de proyectos de DT a costos directos promedio cuando existe una fracción de proyectos a los que se les apoya con negociación y que por lo tanto, incurren en costos $C n$.

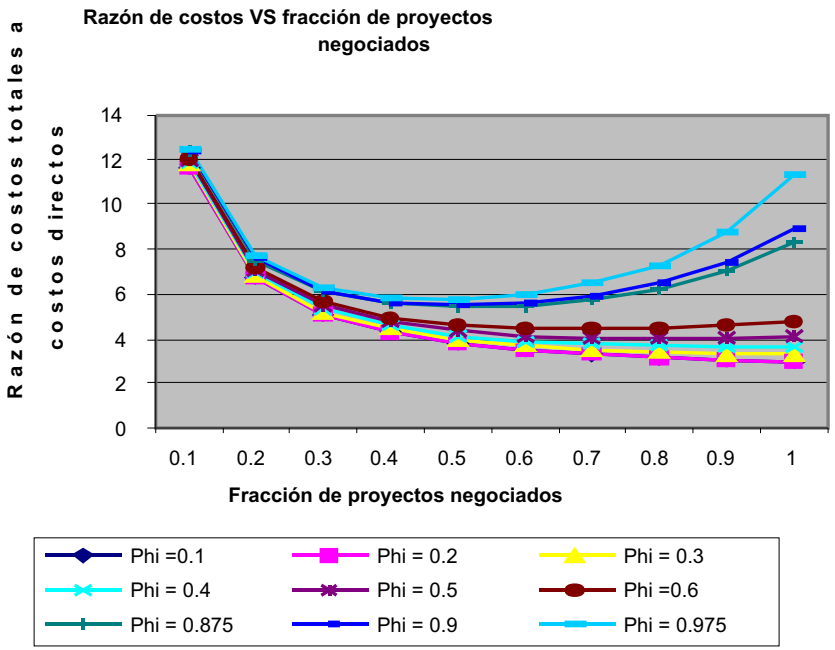

Figura 4. Razón de costos contra fracción de proyectos negociados real

Al evaluar la expresión (13) considerando $\beta=0.8$ y la razón de costos totales a costos directos promedio $\mathrm{R}=\mathrm{C} / \mathrm{CD}=1.5$, obtenemos la curva de la figura 4 , en términos del parámetro $\Psi$, (Phi).

\section{Optimización}

En esta sección para las funciones de costo logradas, obtendremos la fracción de proyectos que debe ser negociada cuando se trabaje con costos mínimos. De la ecuación (13) dado que $\beta$ es constante:

$$
\beta \frac{C}{C D}=\frac{(1+R x)}{\left(-\Psi x^{2}+x\right)}
$$

El óptimo se tendrá cuando la razón de costos sea mínima, aplicando el criterio de la primera derivada:

$$
\frac{d\left(\beta \frac{C}{C D}\right)}{d t}=\frac{\left[\left(-\Psi x^{2}+x\right) R-(1+R x)(-2 \Psi x+1)\right]}{\left(-\Psi x^{2}+x\right)^{2}}
$$

En el óptimo la expresión (15) debe ser igual a cero. Como el denominador es siempre positivo dentro del dominio de $\psi$ y de $x$, igualamos el numerador a cero:

$$
\begin{aligned}
& \left(-\Psi x^{2}+x\right) R-(1+R x)(-2 \Psi x+1)=0 \\
& -\Psi x^{2} R+x R+2 \Psi x-1+2 R \Psi x^{2}-R x=0
\end{aligned}
$$

Resultando la ecuación cuadrática:

$-\Psi R x^{2}-2 \Psi x+1=0$

Que tiene la solución:

$$
x_{\min }=\frac{\left[-1+\sqrt{1+\frac{R}{\Psi}}\right]}{R}
$$

\section{Aplicación}

En un periodo de trabajo donde la razón de costos de proyectos negociados al total de costos directos de proyectos sea $R=1.5$, con ocurrencias de éxito máximo $\beta=0.8$ y mínimo $\alpha=0.1$, tendremos:

$$
\Psi=1-\frac{\alpha}{\beta}=1-\frac{0.1}{0.8}=0.875
$$

Aplicando en la ecuación (15)

$$
X \min =\frac{\left[-1+\sqrt{1+\frac{R}{\Psi}}\right]}{R}=\frac{\left[-1+\sqrt{\frac{1.5}{0.875}}\right]}{1.5}=0.4316
$$


DOI: http://dx.doi.org/10.22201/fi.25940732e.2010.11n4.032

Metodología para valorar las aportaciones de la negociación en la gestión tecnológica de proyectos en un centro de I\&DT universitario

Esto es, que se requiere realizar gestión de la negociación para entre cuatro y cinco proyectos por cada diez proyectos de desarrollo tecnológico $(\mathrm{N}=10)$ desarrollados por el centro para lograr una razón de costos óptima.

Si el total de Costos $C=\$ 1,500,000.00 \mathrm{MN}$ y el total de costos directos de todos los proyectos negociados y no negociados $N^{*} C D=\$ 1,000,000.00 \mathrm{MN}$, aplicando estos valores a la expresión (2), podemos obtener el total óptimo de costos de negociación Cn y el costo de negociación optimo por proyecto y por periodo:

$$
\begin{aligned}
& C=N(C D+x C n)=(1.5)\left(10^{6}\right)= \\
& N C D+N 0.4316 C n)=1000000+N 4.316 C n
\end{aligned}
$$

\section{Entonces}

$$
C n=\frac{1500000-1000000}{4.316}=\frac{500000}{4.316}=115848
$$

El costo promedio de los proyectos que reciben apoyo de negociación resulta de $\$ 115,848.00 \mathrm{MN}$ lo que significa que cada uno de los proyectos negociados representa un $7.7 \%$ del total de costos que es la cantidad de $\$ 1,500,000.00 \mathrm{MN}$. El costo promedio por cada proyecto negociado es $\mathrm{C}_{n}=\$ 11,584.80 \mathrm{MN}$.

Por otra parte, si observamos que en el modelo de circuitos de negociación mostrado en la figura 1, se requiere aproximadamente de siete (7) circuitos de negociación externos para la gestión de cada proyecto, entonces el costo por cada circuito de negociación externo $\left(C n_{C E}\right)$ del modelo de la figura 2, resulta:

$$
C n_{C E}=\frac{\$ 115,488.00}{7}=\$ 16,498.20 \mathrm{MN}
$$

\section{Conclusiones}

Bajo los supuestos de la metodología de costos presentada, los resultados de este trabajo nos permiten concluir que es posible valorar los costos intangibles de negociación que se presentan en la operación conjunta de todos los proyectos de desarrollo tecnológico que se realizan en una organización durante un periodo de tiempo determinado.

La familia de curvas de la figura 4 que muestran la relación entre la razón $\mathrm{C} / \mathrm{CD}$ contra la fracción de proyectos negociados $\mathcal{X}$, representan las curvas de operación real en las que podemos trabajar siguiendo los pasos y los supuestos de la metodología. Idealmente, deberíamos buscar trabajar en la curva que más se acerque al comportamiento de una tasa marginal de sustitución
(MRS, por sus siglas en inglés); esto es, al trabajar con una fracción adicional de proyectos negociados manteniendo la razón de costos. La idea es que los proyectos de desarrollo negociados permitan mantener la operación de la organización dentro de la misma curva de indiferencia; esto es, manteniendo los niveles de utilidad constantes. Esto sucede cuando la razón de cambios de la fracción de proyectos negociados $x$ entre la razón de cambio de "C/CD" permanece constante (Pandey et al., 2006).

Como hemos visto, los circuitos de negociación, como parte de la gestión tecnológica de proyectos, definitivamente generan costos, y por lo tanto, hay que buscar la fracción mínima de proyectos que deben gestionarse a fin de lograr una razón de costos óptima.

Ante la complejidad que significa la adecuada administración de las organizaciones que realizan I\&DT, es indispensable generar competencias de segundo orden dentro de la organización, en el sentido propuesto por Danneels (2002); esto es, se requieren generar las habilidades para identificar, evaluar e incorporar nuevas competencias tecnológicas a la organización, además de las ya existentes. Un primer paso, será reconocer la existencia e importancia de la GT de P, para posteriormente identificar los costos intangibles involucrados y generar metodologías para su cuantificación.

En muchos casos, los costos de gestión y negociación ya se consideran implícitos dentro de los indirectos organizacionales; sin embargo, su cuantificación específica permitirá medir la aportación de estas actividades dentro del valor total de un proyecto.

Al final, el precio de venta de los proyectos debe reportar alguna utilidad a la organización de I\&DT, además de los valores académicos implícitos generados. La medida del costo de la GT de P, indicará indirectamente el valor mínimo porcentual que aportan estas actividades a la organización, en términos del impacto económico o social de los proyectos realizados.

\section{Referencias}

Acosta J., Turrent G., Olín M., González R. A Model for Management of Technology. IEEE International Engineering Management Conference, Albuquerque USA. 2000. Pp. 63-68.

Adams M.E., Day G., Dougherty D. Enhancing New Product Development Performance: An Organizational Learning Perspective. Journal of Product Innovation Management, 15: 404-422. Elsevier Science Inc. 1998.

Ching Ch.L., Yang J. Knowledge Value Chain. Journal of Management Development, 19(9):783-793. MCB University Press. 2000. 
DOI: http://dx.doi.org/10.22201/fi.25940732e.2010.11n4.032

Vega-González L.R.

Danneels E. The Dynamics of Product Innovation and Firm Competences. Strategic Management Journal, 23:1095-1121. Johm Wiley \& Sons Ltd. 2002.

Drucker P.F. The Age of Discontinuity: Guidelines to our Changing Society. New Brunswick USA \& London. Transaction Publishers. 1992.

Drucker P.F. The Discipline of Innovation: the Innovating Enterprise. Harvard Business Review. 2002.

Geistauts G.A., Backer E.R., Eschenbach T.G. An Integrated Technology Management Model, 91. Portland International Conferences on Management of Engineering Technology, IEEE. 1992. Pp. 742-744.

Hidalgo A. Mecanismos de excelencia para la transferencia de la tecnología desarrollada en la universidad a la industria. Anales del seminario iberoamericano sobre tendencias modernas en Gerencia de la Ciencia y la Innovación Tecnológica. IBERGECIT'97. 1997. Pp. 227-243.

Hindle K., Yencken J. Public Research Commercialization, Entrepreneurship and New Technology Based Firms: an Integrated Model. Technovation, 24:793-803. 2004.

Jacob W.F., Kwak Y.H. In Search of Innovative Techniques to Evaluate Pharmaceutical R\&D Projects. Technovation, 23:291-296. Elsevier Ltd. 2003.

Kerzner H. Project Management, a Systems Approach to Planning, Scheduling and Controlling. Third Edition. New York. Van Nostrand Reinhold. 1989. Pp. 711-758.
Knoepfel H., Berger R. Project Cost Management in Project Management in Progress: tools \& Strategies for the 90's. M.C. Grool et al. (Editors). North Holland. Elsevier Science Publishers. 1986. Pp. 239-246.

Lara R.F. Informe de trabajo de la Dirección del Centro de Ciencias Aplicadas y Desarrollo Tecnológico: periodo 1998-2005, UNAM. 2005.

Lieb E.B. How Many R\&D Projects to Develop? IEEE Transactions on Engineering Management, 45(1):73-77, February 1998.

Linn R.J., Zhang W., Li Z.Y. An Intelligent Management System for Technology Management. Computers \& Industrial Engineering, 38:397-412, Elsevier Science Ltd. 2000.

Lundvall B.A. National Systems of Innovation: Towards a Theory of Innovation and Interactive Learrning. London. Pinter Publishers. 1992.

Medellín C.E. Transferencia de tecnología de la universidad al sector productivo. Anales del Seminario-Taller Iberoamericano de Actualización en Gestión Tecnológica IBERGECIT' 96, 1996. Pp72-91.

Pandey M.D., Nathwany J.S., Lind N.C. The Derivation and Calibration of Life-Quiality Index (LOI) from Economic Principles. Structural Safety, 28(4):341-361. Elsevier. 2006.

Porter M.E. Competitive Advantage: Creating and Sustaining Superior Performance. The Free Press. EUA. Mc. Millan Inc. 1997.

Tribe K. Reading Trade in the Wealth of Nations. History of European Ideas, 32:58-79. 2006.

\section{Semblanza del autor}

Luís Roberto Vega-González. Es ingeniero mecánico electricista por la Facultad de Ingeniería de la Universidad Nacional Autónoma de México (UNAM). Obtuvo la maestría en ingeniería de sistemas en el área de planeación por la División de Estudios de Posgrado de la Facultad de Ingeniería UNAM y la maestría en administración de las organizaciones por la División de Estudios de Posgrado de la Facultad de Contaduría y Administración de la UNAM. Se especializó en gestión tecnológica por el Centro de Innovación Tecnológica y la FCy A de la UNAM. Durante más de veinte años colaboró en diversas firmas nacionales e internacionales desarrollando proyectos de ingeniería e integración tecnológica en las áreas de instrumentación, control y potencia. Desde 1993, es académico de tiempo completo en la UNAM, donde ha colaborado en el Centro para la Innovación Tecnológica, el Instituto de Ingeniería y el Centro de Instrumentos. Actualmente es coordinador de vinculación y gestión tecnológica del Centro de Ciencias Aplicadas y Desarrollo Tecnológico (CCADET). Ha conducido negociaciones, convenios, contratos y realizado actividades de administración tecnológica para cerca de dos centenares de proyectos en la UNAM. Es autor de aproximadamente un centenar de diferentes productos académicos en sus diferentes modalidades en los temas de su especialidad. 